\title{
CARACTERIZACIÓN ENERGÉTICA BASADA EN EL ESTÁNDAR ISO50001 DE UNA PLANTA DE PRODUCCIÓN DEL SECTOR AGROINDUSTRIAL
}

\section{ENERGY CHARACTERIZATION BASED ON THE ISO50001 STANDARD OF A PRODUCTION PLANT IN THE AGROINDUSTRIAL SECTOR}

\section{ALEXYS MORALES MEDRANO',}

Monómeros Colombo Venezolanos S.A.,

Barranquilla, Colombia.

GONZALO ROMERO GARCIA ${ }^{2}$, ing.

Facultad de Ingeniería, Universidad Francisco

de Paula Santander, Cucuta, Colombia

DORA CLEMENCIA VILLADA CASTILLO ${ }^{3}$,

Facultad de Ingeniería, Universidad Francisco

de Paula Santander, Cucuta, Colombia

\section{RESUMEN}

Las actividades de caracterización tecnológica, energética y organizacional desarrollada en una empresa productora de fertilizantes

\section{ALEXYS MORALES MEDRANO,}

alexismorales64@gmail.com,

Monómeros Colombo Venezolanos S.A., Barranquilla, Colombia.

2 GONZALO ROMERO GARCIA

ing.gonzaloromerogarcia@gmail.com,

Facultad de Ingeniería, Universidad Francisco de Paula Santander, Cucuta, Colombia

3 DORA CLEMENCIA VILLADA CASTILLO

Facultad de Ingeniería, Universidad Francisco de Paula

Santander, Cucuta, Colombia

en Barranquilla, se fundamentaron en los requerimientos establecidos en la Norma ISO 50001:2018, la cual fue auspiciada por UNIDO (Organización de Naciones Unidas para el Desarrollo Industrial) y emitida por la organización ISO el 6 de junio de 2011 a nivel mundial, acogida en Colombia por ICONTEC para el 20 de enero de 2012, posteriormente actualizada el 19 de septiembre del 2018 y se conoce actualmente como norma ISO 50001:2018. En cuanto a potencial de ahorro de la planta, el potencial de ahorro asociado a 
buenas prácticas de manufactura en la que el consumo no está asociado a la producción se puede reducir en un $9,5 \%$, lo que se traduce en 1408 kWh/día. Para el consumo de energía eléctrica existe una producción critica (PCRIT) de acuerdo con la tecnología de la planta, calculada en $1400 \mathrm{Tm} /$ día y una producción promedio (PPROM) de la planta de $1304 \mathrm{Tm} /$ día, el cual se traduce en 3623 kWh/día.

\section{PALABRAS CLAVE}

Caracterización energética, indicadores energéticos, norma ISO 50001.

\section{ABSTRACT}

The technological, energetic and organizational characterization activities developed in a fertilizer production company in Barranquilla, were based on the requirements established in the ISO 50001:2018 Standard, which was sponsored by UNIDO (United Nations Industrial Development Organization) and issued by the ISO organization on June 6, 2011 worldwide, accepted in Colombia by ICONTEC on January 20, 2012, subsequently updated on September 19, 2018 and is currently known as ISO 50001:2018. In terms of the plant's savings potential, the savings potential associated with good manufacturing practices in which consumption is not associated with production can be reduced by $9.5 \%$, which translates into $1408 \mathrm{kWh} /$ day. For electrical energy consumption there is a critical production (PCRIT) according to the plant technology, calculated at $1400 \mathrm{Tm} /$ day and an average production (PPROM) of the plant of $1304 \mathrm{Tm} /$ day, which translates into $3623 \mathrm{kWh} /$ day.

\section{KEY WORDS:}

Energy characterization, energy indicators, standard ISO 50001.

\section{INTRODUCCIÓN}

La gestión energética representa un factor estratégico que incide en la productividad y el posicionamiento empresarial. Así mismo, la alta competitividad en los precios de los productos ha obligado a las industrias a buscar alternativas que les permitan reducir los costos de producción y sugieran un uso apropiado de los recursos con que disponen. Una de estas alternativas es minimizar el costo del consumo energético, mediante un uso racional y eficiente de la energía, de esta manera se hace necesario contar con un sistema de gestión energética que pueda garantizar la competitividad de la empresa en el mercado, y que contribuya a mejorar la eficiencia energética de manera continua.

Por lo anterior, es necesario realizar un estudio que permita identificar los posibles potenciales de ahorro en una planta productora de fertilizantes y, así, evaluar la eficiencia con la que se administran y distribuyen todos los tipos de energía asociados al proceso productivo. Una vez realizado el análisis del consumo real, se puede implementar un sistema de gestión integral, que se pueda traducir en opciones de mejora a corto, mediano y largo plazo.

Dada la creciente competitividad de las industrias es de principal importancia analizar los procesos de mayor consumo energético de una empresa en búsqueda de generar una línea de producción amigable con el medio ambiente y la reducción de costos (Jovanović \& Filipović, 2016)

Dentro del campo de producción energética, se persigue el desarrollo de nuevos procesos, producción y logística; pocas veces se considera un plan de mejoramiento de la eficiencia energética con el objetivo de obtener una reducción de costos energéticos (Finnerty et al., 2018) donde no solamente se tiene un beneficio económico sino también una predicción cuantificable en la mitigación del cambio climático (McKane et al., 2017) 
Colombia desde el año 2008 se acoge a la propuesta presentada por EE. UU. y Brasil en donde se propone bajo la supervisión de expertos energéticos, un plan de cumplimiento a las prácticas empresariales que van en pro de convertir cada proceso industrial en uno eficiente con respecto a los estándares mundiales (Dzene et al., 2015). La gestión de la energía necesita obedecer a las directrices de mejoramiento continuo del desempeño energético, el cual da como resultado el aumento de la productividad, mayor seguridad operacional y autofinanciamiento a partir del aprovechamiento de los recursos (Campos \& Prias, 2013). En Colombia existe un verdadero problema ambiental, que afecta a gran parte de la población ya que la producción energética en dicho país depende principalmente de la afluencia de sus ríos, el cual se ve duramente golpeado por los fenómenos naturales que trae consigo el cambio climático (Ríos-Ocampo et al., 2019).

La cuantificación de los elevados requerimientos de energía obliga a las empresas de este país a apuntar al desarrollo de métodos eficientes que aumenten los estándares de vida de la sociedad (Pardo Martínez \& Alfonso Piña, 2016). El desarrollo de energías renovables es un paso necesario para mitigar el cambio climático, las cuales apuntan a energía abundante, económica y limpia, en donde la actitud que toma la sociedad es muy importante y está ligada por los costos percibidos y la planificación a largo plazo para la implementación de dichas nuevas tecnologías (Ylipulli et al., 2014). De acuerdo con estudios realizados por la unidad de planeación minero-energética (UPME), para el año 2050 la demanda energética del país, en el peor de los casos, será $62.8 \%$ mayor a lo que es en la actualidad, una cifra que se deberá de suplirse con los vastos recursos de energías renovable potenciales presentes en el país.
Mirando al futuro, si se sigue utilizando los combustibles fósiles como la fuente primaria de producción energética se proyecta una concentración de 1000 ppm más del doble de lo que está presente en nuestra atmosfera. Bajo los mismos escenarios, la temperatura media global de la superficie aumenta de tres a cinco grados en relación a un siglo atrás (Kibria et al., 2019)

En una proyección realizada en el año 2016 por la administración de información energética de EE. UU., las emisiones mundiales de $\mathrm{CO}_{2}$ se proyectan a 32.300 millones de toneladas métricas en 2012, alcanzando 43.200 millones de toneladas métricas en 2040 (Kibria et al., 2019) we examine the relationship between fossil fuel share in the energy mix and real income using a panel of 151 countries from 1971 to 2013 . A cursory examination of the data suggests that there may exist a polynomial relationship between fossil fuel share and income, a phenomenon that we coin as energy mix Kuznets curve (EMKC. El creciente desarrollo industrial ha generado una concientización por parte de las empresas con respecto al manejo de sus energías primarias en los procesos (Iwata, 2006).

En este articulo se presenta la información sobre el diagrama organizacional, diagrama energético productivo, matriz energética y procesos de la planta, estableciendo un punto de partida que permita la óptima implementación de un Sistema de Gestión Integral de la Energía con miras a certificación con la norma ISO 50001:2018.

\section{METODOLOGÍA}

\subsection{DESCRIPCIÓN GENERAL DE LA GESTIÓN ENERGÉTICA}

La gestión de la energía se define como la coordinación proactiva, organizada y sistemática de la adquisición, conversión, distribución y uso de la energía, que busca cumplir con los requisitos establecidos por la normativa, 
basándose en los objetivos ambientales y económicos. Se centra en la planificación y ejecución de objetivos relacionados con la energía, como la conservación de recursos, la reducción de la huella de carbono y el ahorro de costos (Kanneganti et al., 2017)

Un sistema de gestión de energía (SGEn) es un marco documentado que define los objetivos, políticas, procedimientos y los procesos mediante los cuales se mantienen y mejoran. Hasta hace poco, la eficiencia energética se consideraba solo durante la modificación de una planta de producción o al realizar nuevas inversiones de capital. Pero durante el funcionamiento normal, la eficiencia energética o la conservación ya no es una prioridad de gestión (Gordić et al., 2010).

Todo sistema de administración energética tiene como requisito fundamental la mejora continua, la cual puede lograr por medio del ciclo PlanificarHacer-Verificar-Actuar (PHVA). El ciclo PHVA consta de cuatro etapas, a saber, Planificar, Hacer, Verificar y Actuar, que se pueden usar en sistemas para ayudar a las instalaciones a abordar los procesos para encontrar soluciones a los problemas. Una vez finalizada la etapa Actuar, el ciclo vuelve a la etapa Planificar, lo que le da al ciclo PHVA las características de mejora continua (Kanneganti et al., 2017)

\subsection{INDICADORES ENERGÉTICOS}

Diagrama organizacional: mediante este diagrama se representa gráficamente la estructura organizacional de una compañía, definiendo todos los procesos sistemáticos vinculados a las tareas y actividades que pretende realizar (Dąbrowska, 2018).

Diagrama energético productivo: este diagrama se basa en la estructura del proceso productivo de la compañía, presentando las entradas de energéticos primarios y secundarios que son ocupados en el proceso productivo, incluyendo los equipos que se encargan de la transformación de estos energéticos (Campos \& Prias, 2013).

Balance energético: el balance energético se genera a partir del diagrama energético productivo teniendo en cuenta los principales procesos y consumos energéticos primarios y secundarios, incluyendo las cantidades y los tipos de energéticos empleados. Con esta información, se desarrolla una tabla en la que se relaciona la transformación de estos energéticos y la eficiencia de los equipos que se encargan de realizar ese proceso (Campos \& Prias, 2013).

Usos significativos de energía (USEn): los Usos Significativos de la energía corresponden a aquellos procesos de mayor impacto en el consumo de energía y se seleccionan con base en la información proveniente del balance energético. Esto se consigue mediante un Diagrama de Pareto en el cual se tienen en cuenta los consumos de todos los equipos, incluyendo la energía consumida por los equipos transformadores de energía primaria. Para la selección de los equipos o procesos del Pareto, se escogen el $20 \%$ de los equipos que representan el $80 \%$ del consumo (Campos \& Prias, 2013).

Variables significativas: las variables de mayor influencia en el consumo energético se pueden clasificar en variables estáticas, variables dependientes de la operación y variables dependientes del mantenimiento. Las primeras corresponden a aquellas que afectan el consumo energía, pero son invariables en el tiempo. Las segundas corresponden a las variables que pueden ser controladas durante el proceso. Las terceras corresponden a las variables que pueden controlarse con actividades de mantenimiento (Campos \& Prias, 2013).

Indicadores de desempeño: son herramientas que ofrecen información cuantitativa con relación al estado de la organización tales como 
cumplimiento de logros, programa, actividad o proyecto.

Línea base: la línea base energética es un indicador energético que se genera relacionando el consumo energético con la producción, y proporciona una base de comparación para el desempeño energético. Esta se puede normalizar utilizando variables que afecten el desempeño energético, como lo son la temperatura y presión ambiental, etc. (Campos \& Prias, 2013).

Para la construcción de esta línea se debe primero definir el periodo de la base, este puede ser anual, mensual, semana o diario. Teniendo esto, se define la muestra de datos mínima y se definen las variables que van en la línea base. Para definir esta muestra mínima, se utiliza la ecuación (1).

$$
n_{0}=\frac{Z^{2} * c v^{2}}{e^{2}}
$$

Donde $Z$ es el valor de distribución normal respecto a los datos tomados, el nivel esperado y cv es el coeficiente de varianza, definido como la desviación estándar de las lecturas dividida por la media. Luego de esto, se toman los datos y se verifica en coeficiente de variación; para finalmente filtrar los datos hasta elevar el $r^{2}$ para la muestra mínima. Se puede aplicar el ajuste de población finita mediante la ecuación (2). Este ajuste entrega un rango de datos requeridos para cada periodo posible.

$$
n=\frac{n_{o} * N}{n_{0}+N}
$$

Aplicando un modelado lineal a los datos de producción y consumo seleccionados se obtiene la ecuación (3).

$$
E=m * P+E_{o}
$$

Donde $E$ es el consumo de energía, $P$ es la producción y $E_{O}$ es el consumo no asociado a la producción. Esta energía viene dada por todos los equipos que consumen energía necesaria para el proceso y no está asociado directamente a la producción. Por ejemplo, el sistema de iluminación de la planta, el sistema de aire acondicionado de las oficinas etc.

Índice de consumol índice energético nos muestra como varia el consumo de energía en función de la producción. Aquí podemos observar si la muestra trabaja en zonas de bajo índice de consumo, de índice medio o zonas de alto índice de consumo. Además de esto, nos revela la influencia del factor de carga del área, línea o equipo en el consumo de energía por unidad producida (Campos \& Prias, 2013). Para obtener este índice, se divide el consumo de energía dado por la línea base entre la producción como se observa a continuación:

$$
I C=\frac{E}{P}
$$

$$
\begin{aligned}
& \frac{E}{P}=m+\frac{E_{o}}{P} \\
& I C=m+\frac{E_{o}}{P}
\end{aligned}
$$

Con esta ecuación, se genera un gráfico en el cual se puede observar las 3 zonas anteriormente descritas, y de esta forma identificar en cual de esta se encuentra la muestra. Este grafico muestra una figura exponencial decreciente y el valor en que se empieza a presentar la asíntota es conocido como taza de producción critica. Esta taza es el valor de producción mínimo que se desea buscar para que el valor del IC tienda a ser invariante.

Para el cálculo de la taza de producción crítica se debe realizar una tabla de datos del consumo y producción reales en la unidad de tiempo seleccionada para la línea base. Posteriormente, 
agregar una columna calculando el IC real y otra calculando el IC teórico con la ecuación (6) para cada dato de producción. Seguido a esto, agregar una columna calculando la pendiente dividiendo la energía calculada mediante la línea base entre el cuadrado de la producción; esto para cada valor de producción. Teniendo todo esto, se ordena la tabla con la producción descendente y se crea una columna con una suma acumulativa de las pendientes y se gráfica.

La obtención de este punto es de gran importancia debido a que a partir de él se puede determinar un potencial de ahorro por trabajar a tazas iguales o superiores al punto de quiebre. Para esto, calculamos el IC promedio de la empresa hasta antes de la nueva planeación de producción y calculamos la producción promedio en este mismo periodo. Teniendo estos dos datos junto al punto de quiebre obtenido con la metodología anteriormente descrita, se calcula el potencial de ahorro mediante la ecuación (7).

$$
A p=P_{\text {prom }} *\left(I C_{\text {prom }}-I C_{\text {crit }}\right)
$$

Indicador base 100: el índice base de eficiencia 100 (Base 100) es una herramienta de gestión energética que permite evaluar el resultado como comportamiento del consumo de energía medido a lo largo del tiempo de operación de la planta, respecto a los valores teóricos de consumo de energía calculados con la línea base (Campos \& Prias, 2013).

\section{Indicador de tendencia de desempeño} CUSUM: al igual que los indicadores anteriormente presentados, el indicador CUSUM se utiliza para realizar una evaluación al rendimiento energético de la compañía. A diferencia de los anteriores, este indicador parte de la selección de un periodo base de comparación y los resultados del CUSUM serán medidos solo frente a ese periodo de referencia.
Este indicador presenta una tendencia del desempeño energético a partir de la suma acumulativa de la diferencia del consumo energético real menos el consumo teórico calculado mediante la línea base. Si los resultados de la tendencia son mayores a cero, nos encontraremos en una zona de no conformidad. Por el contrario, si los resultados son negativos, nos encontraremos una zona de conformidad y buen desempeño respecto al periodo base (Campos \& Prias, 2013).

\section{RESULTADOS}

\subsection{Análisis de indicadores de desempeño energético.}

Para el análisis de los indicadores de desempeño energético se utilizarán indicadores como la línea base y línea meta, e índice de consumo real vs índice de consumo teórico que indican potenciales de ahorro por buenas prácticas y por planeación respectivamente. Luego se presentarán indiciadores de desempeño como el Índice de Eficiencia Base 100 y el de tendencia acumulada CUSUM. Esta sección se centra en el análisis de los indicadores, arriba mencionados, para el consumo de energía eléctrica y energía térmica en forma de gas natural, directamente relacionado a la producción de la planta.

\subsection{Gráfica Energía vs Producción}

De acuerdo con los datos suministrados por el equipo de MCV en el formato FD02, y luego de haber aplicado el primer filtro de los gráficos de límite de control, se obtiene la línea base que se observa en la Figura 1 donde se obtiene una correlación de con una ecuación de línea base, con una estructura base de la ecuación (8), cuyos valores se muestran en la ecuación (9).

$$
\begin{aligned}
& E_{t}=m * P+E N A \\
& E_{t}=11,027 * P+14440
\end{aligned}
$$


Donde se identifica que el consumo energético por unidad de tonelada producida en una tasa de 11,027 kWh/TM, además de que se evidencia los altos niveles de ENAP (Energía No Asociada a la Producción), relacionados a procesos administrativos y demás que no influyen directamente en la producción de la planta.

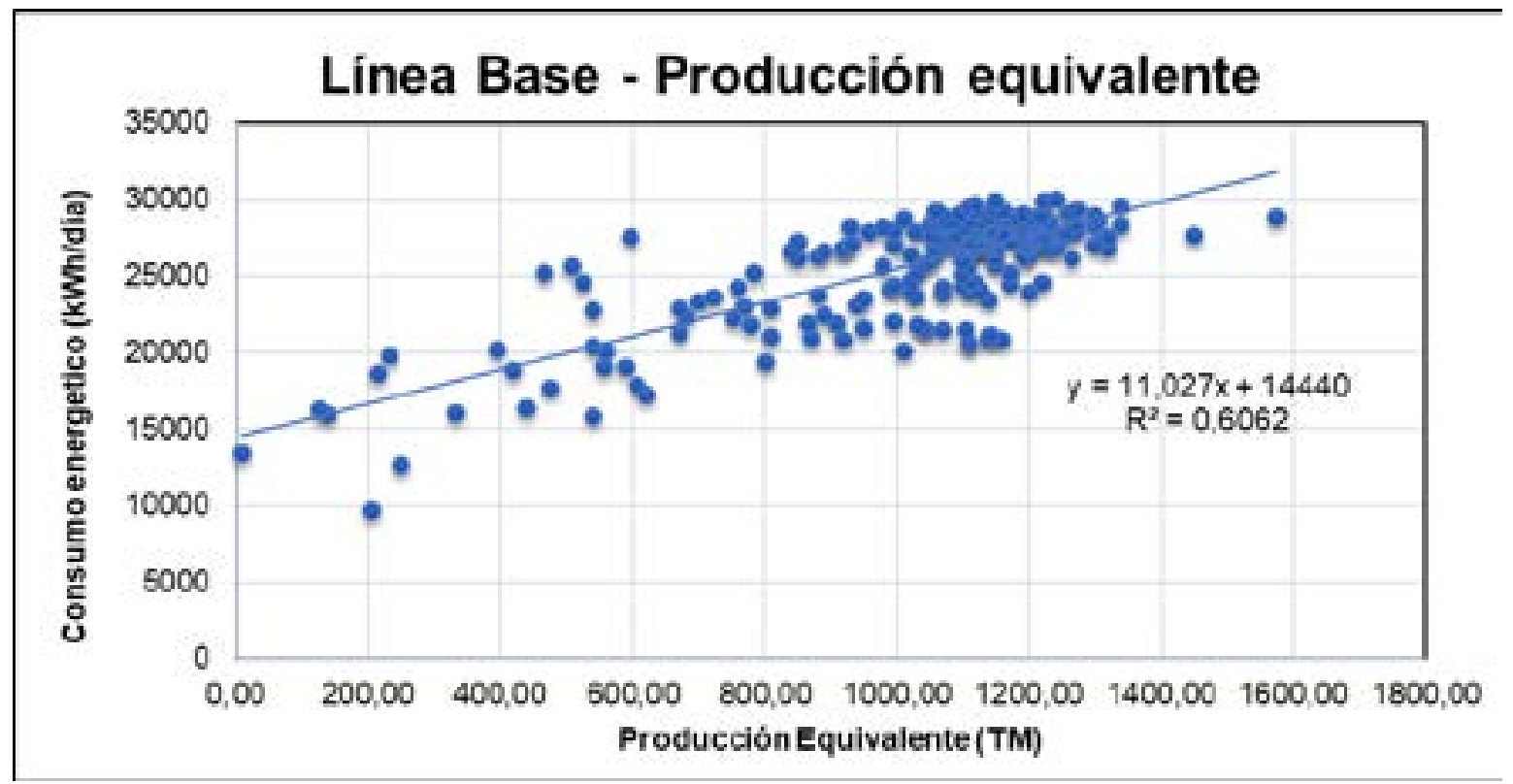

Figura 1. Línea Base de consumo energético.

Teniendo en cuenta que la línea base representa un consumo energético nominal para la operación de la planta, a partir de los registros históricos de producción y consumo energético, se define que los datos por encima de la línea base representan puntos de bajo desempeño energético, mientras que los datos por debajo de la línea base representan.

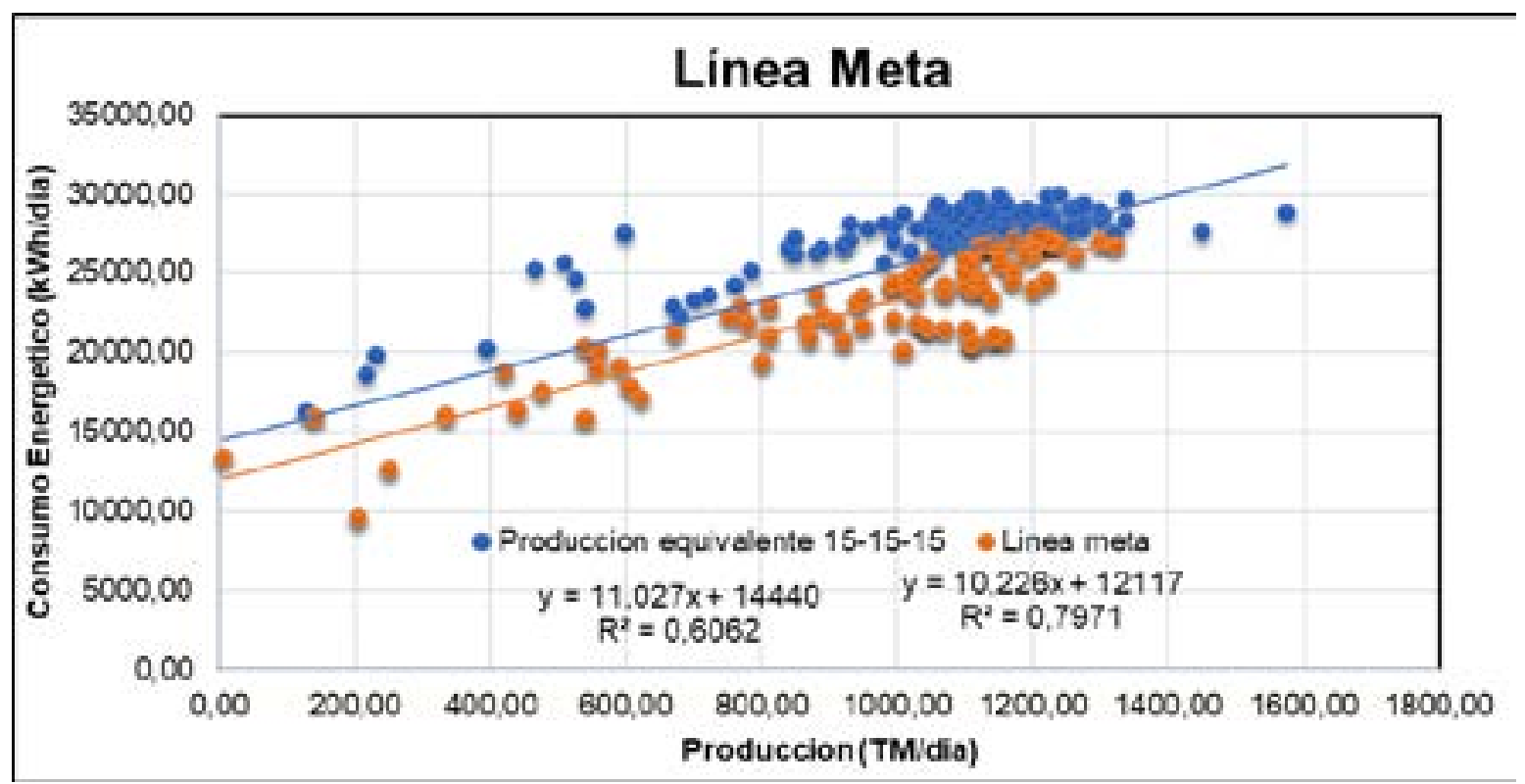

Figura 2. Línea Meta de Consumo Energético 
A partir de los datos de buen desempeño energético, señalados con marcadores de color rojo, se construye una Línea Meta, la cual establecerá un potencial de ahorro de consumo energético asociado a las buenas prácticas de manufactura, sin necesidad de realizar cambios tecnológicos en la infraestructura de la planta, este potencial de ahorro de consumo energético es determinado mediante la diferencia de las ENAPs de la línea base y la línea meta, este porcentaje de ahorro se muestra en la Tabla 1.

Tabla 1. Potencial de ahorro energético por BPM.

\begin{tabular}{lll}
\hline ENAP Línea Base & 14440 & kWh \\
ENAP Línea Meta & 12117 & kWh \\
Porcentaje de ahorro & $9.5 \%$ & \\
\hline
\end{tabular}

El índice de consumo energético se define como la relación entre la energía consumida y el valor de la producción obtenida mediante

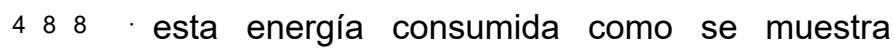
en la ecuación (17), es un indicador que de desempeño energético que se analiza mediante la comparación del índice de consumo real con en el índice de consumo teórico, ambos graficados contra la producción de la planta. EI índice de consumo real se construye mediante los datos de consumo energético y producción real como se muestra en la ecuación (10).

$$
I C_{\text {Real }}=\frac{E_{\text {Real }}}{P}
$$

Mientras que el índice de consumo teórico se construye mediante la ecuación (11)

$$
I C_{\text {teorico }}=\frac{E_{\text {teorico }}}{P}
$$

El consumo energético teórico utilizado en la ecuación (12) es calculado utilizando la ecuación (9) de línea base ajustada, por lo que reemplazando la ecuación (9) en (11) se obtiene la ecuación (12) para calcular el índice de consumo teórico.

$$
I C_{\text {teorico }}=11,027+\frac{14440}{P}
$$

La comparación del índice de consumo real vs índice de consumo teórico se observa en la Figura 3, donde los datos en azul representan el índice de consumo real, mientras la línea continua en representa el índice de consumo teórico.

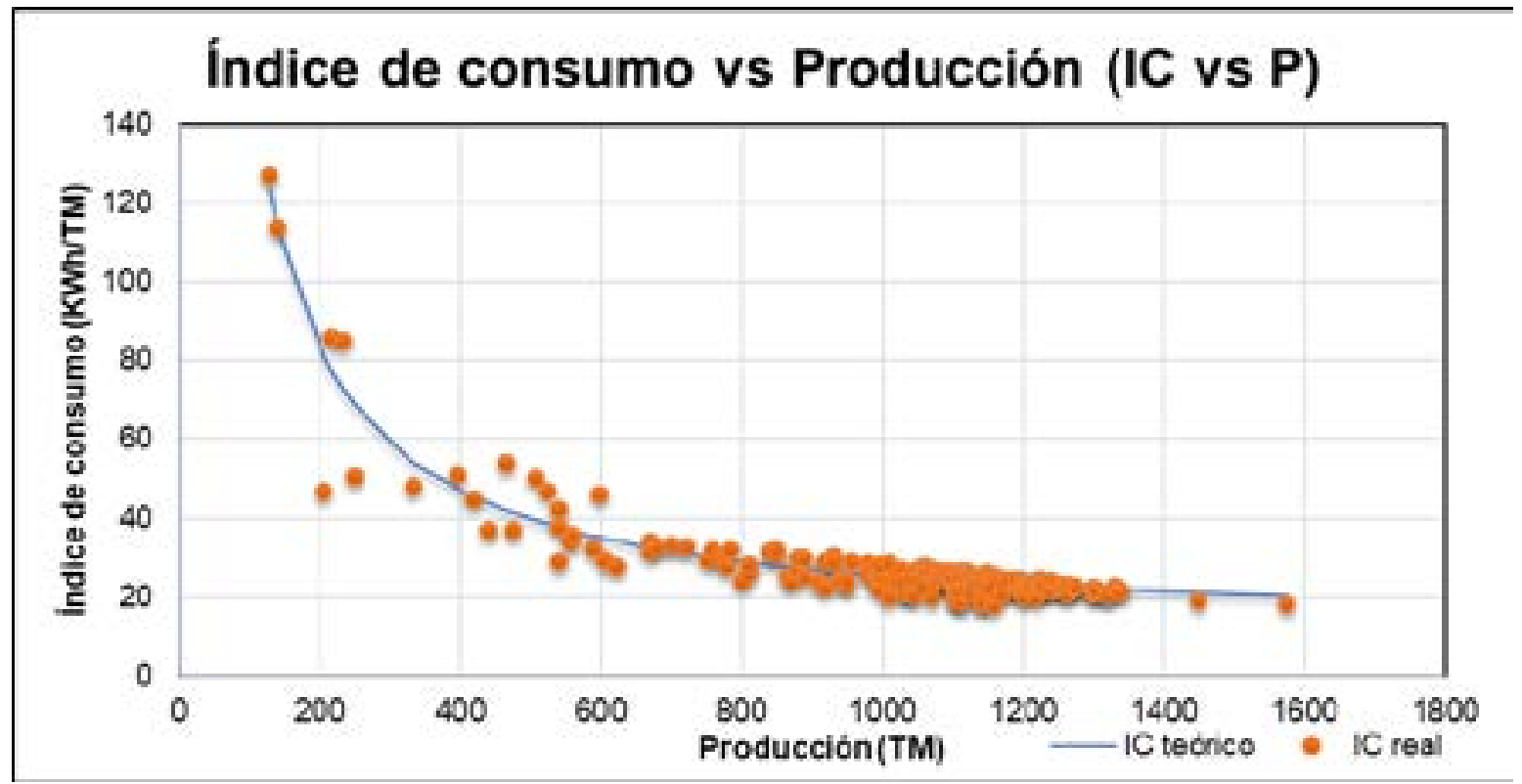

Figura 3. Índice de Consumo Teórico vs Índice de Consumo Real. 
En la Figura 3, se observa que el índice de consumo real de la planta varia normalmente entre 20 y $60 \mathrm{kWh} / \mathrm{TM}$, sin embargo, se encuentran puntos de alto índice de consumo teórico que representan un mal manejo del recurso energético, se identifican valores claves como la producción promedio de 1034 TM/día y un consumo energético promedio de 25852,6 kWh/día. Además, se permite identificar la tasa de producción diaria, a la cual la planta debería operar, de tal manera que el índice de consumo no presente variaciones significativas, esta tasa de producción se conoce como tasa de producción crítica y se identifica en 1400 TM/ día, o 58,33 TM/h.

\subsection{Gráfico de tendencia o CUSUM}

El grafico de tendencia acumulada o CUSUM permite realizar un monitoreo en la tendencia de consumo de energéticos para la empresa, inicialmente para electricidad, con respecto a un periodo base.

Para el análisis del consumo eléctrico de la planta. se tomará como periodo base el periodo del año 2015, para así monitorear la tendencia del consumo en el mismo año, identificando posibles periodos de tiempo donde se presente buen desempeño energético en la empresa, o si es el caso, identificar periodos de tiempo donde se encuentre mal desempeño energético con el fin de establecer oportunidades de mejora.

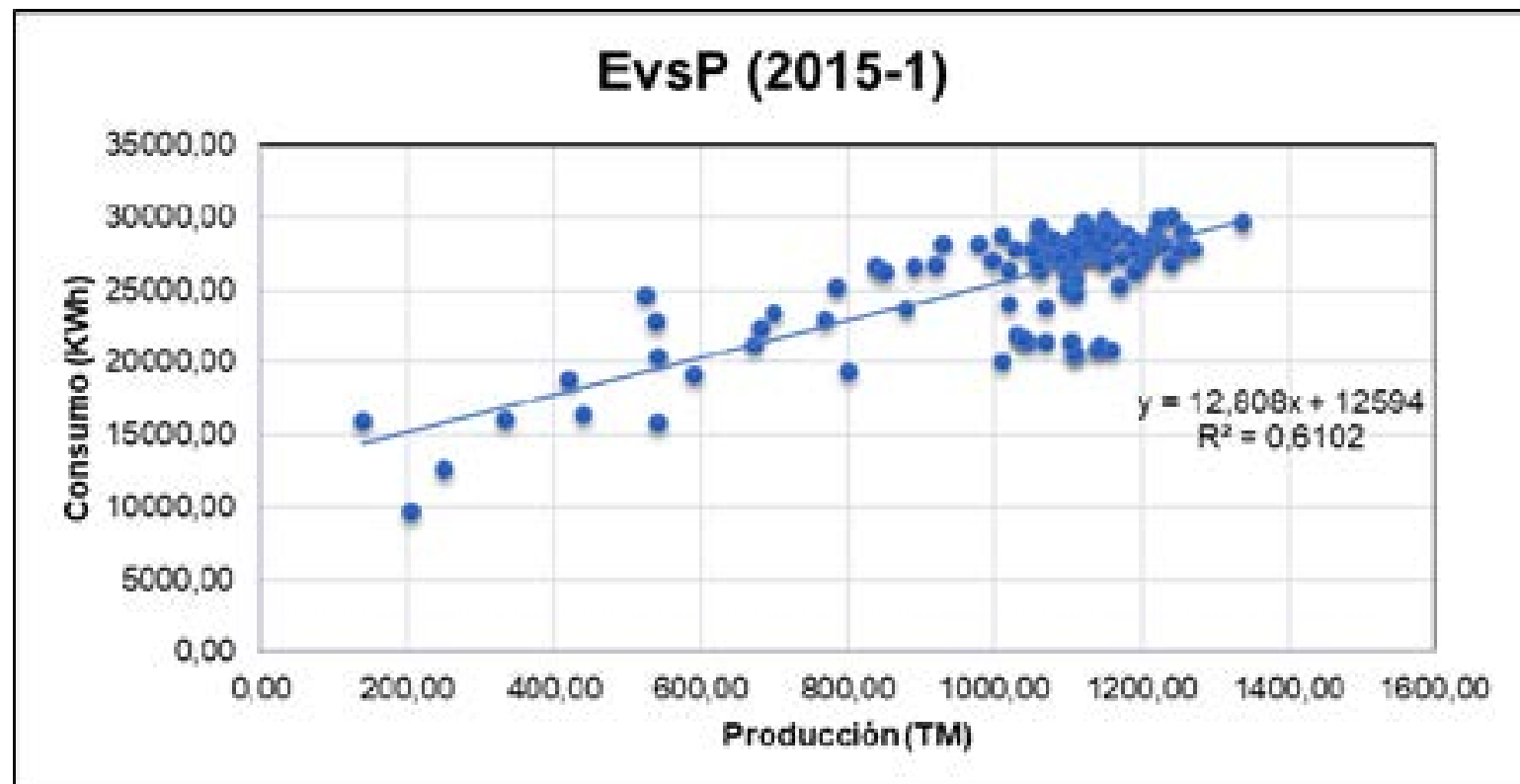

Figura 4. Consumo De Electricidad Para El Año 2015.

En la Figura 5, se observa que durante la mitad del periodo de Agosto se mantuvo un comportamiento estable, con una tendencia hacia el ahorro de energía eléctrica hasta finales de Agosto, donde se presenta una variación en la pendiente que cambia de la tendencia del ahorro hacia el desahorro, adicionalmente se pueden identificar periodos adicionales donde hay variación de la pendiente en el consumo, aun así a partir de este momento las tendencias se inclinan hacia el desahorro energético. 


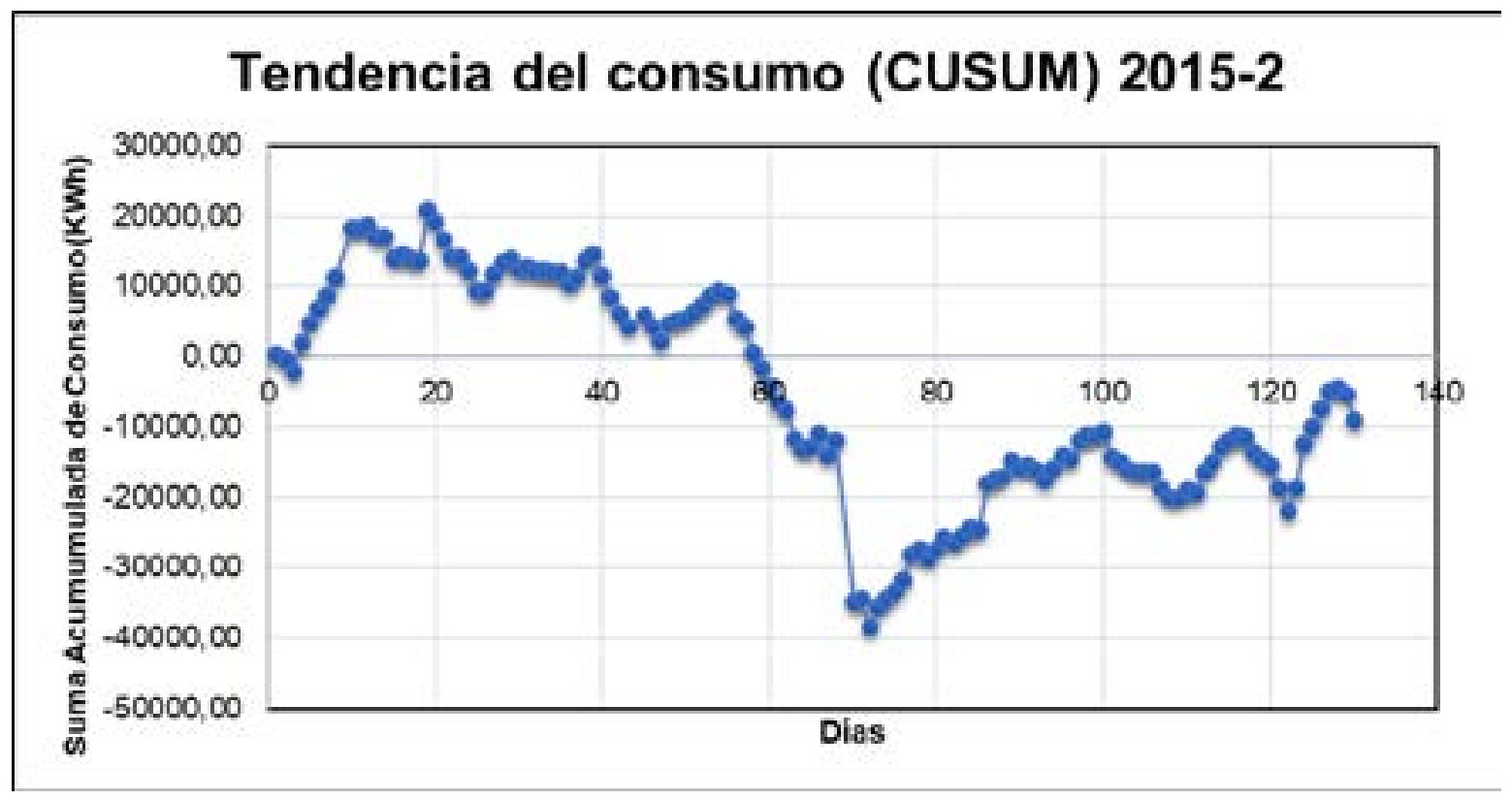

Figura 5. Tendencia acumulada del Consumo de Electricidad para el año 2015.

\section{4 Índice de Eficiencia Base 100}

El índice de eficiencia base 100 es una herramienta de gestión energética, que permite evaluar el comportamiento de los resultados de consumo energético medidos durante un periodo de tiempo operativo de la planta respecto a los valores teóricos de consumo energético calculados mediante la línea base. El índice de eficiencia base 100 se calcula mediante la siguiente ecuación:

$$
\text { Base } 100=\frac{E_{\text {teorico }}}{E_{\text {Real }}} \times 100 \%
$$

Este indicador genera alertas en cuanto a las variaciones positivas o negativas en la eficiencia energética del proceso, así facilitando el análisis y propuesta de planes de acción en función de mejoras energéticas, permitiendo la interacción analítica entre la producción y el consumo energético, apuntando a un mejor desempeño energético de los procesos de la planta.

El índice de eficiencia base 100 puede expresar dos intervalos de resultados B100 $<100 \%$ y B100 $\geq 100 \%$. Para el caso cuando el índice es mayor a $100 \%$ se traduce en un buen desempeño energético, dado que el consumo eléctrico real fue menor al consumo eléctrico esperado, por lo tanto, en la Figura 6 los puntos por encima de la línea límite de color rojo se consideran datos de buen desempeño energético ubicados en la zona de eficiencia energética de la planta. Caso contrario, cuando el índice de eficiencia es menor a $100 \%$, los datos se ubican por debajo de la línea de color rojo y se indica que los datos pertenecen a una zona de ineficiencia energética de la planta. Sin embargo, para el análisis de la planta en la zona de eficiencia energética se presentan un pico de alta eficiencia con un comportamiento aleatorio, por lo que se dice estos puntos pico ubicados en la zona de eficiencia energética no son resultado de mejoras en el sistema de gestión energética de la empresa. 


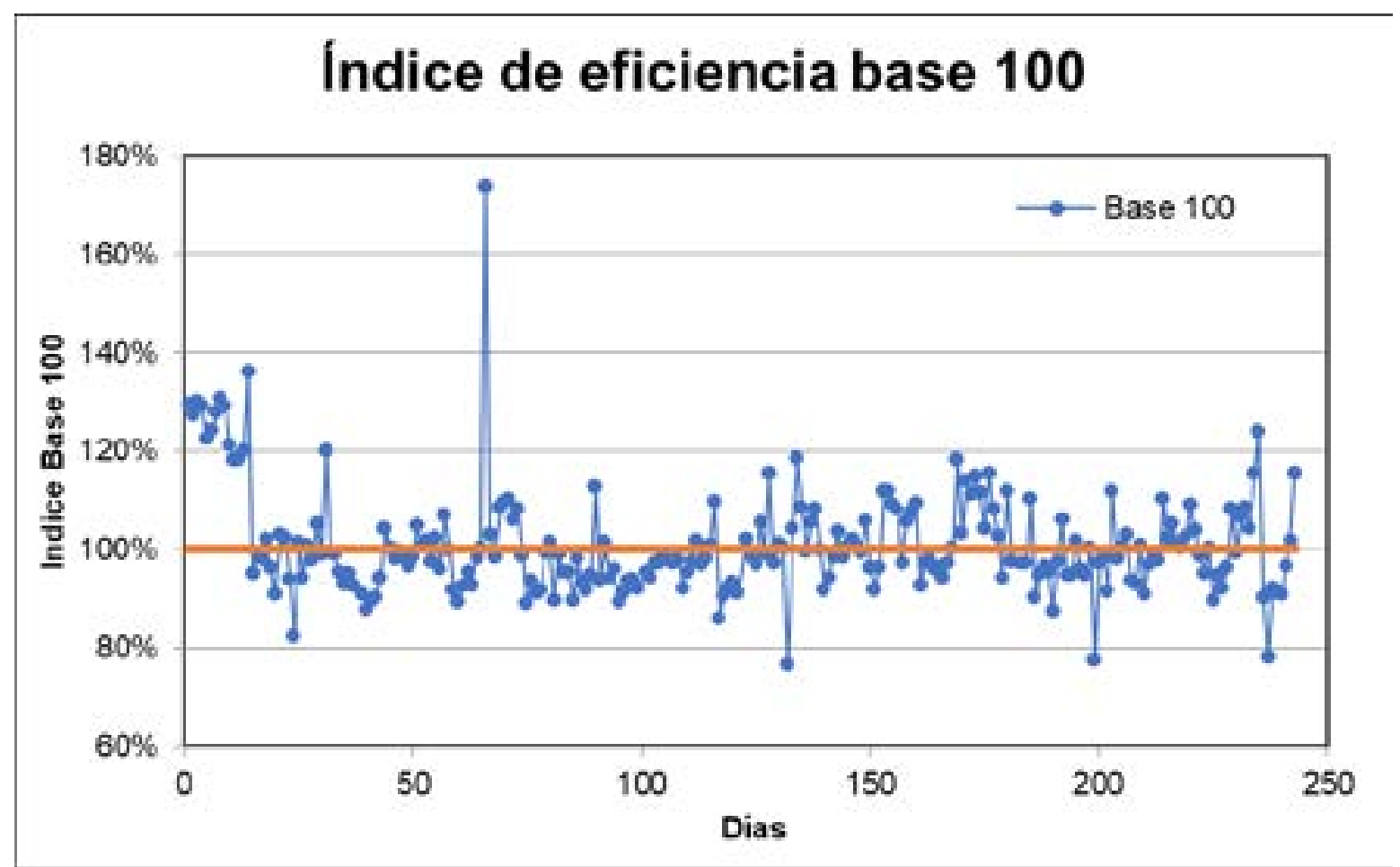

Figura 6. Índice de eficiencia Base 100 para energía Eléctrica.

\section{CONCLUSIONES}

A partir de los resultados en cada una de los aspectos evaluados en la etapa preliminar de revisión energética en la planta, los gestores energéticos se consideró que para la Implementar un Sistema de Gestión Energética según la norma ISO 50001:2018 en la compañía atendiendo a los usos significativos de energía en la planta, es primordial el monitoreo y control de indicadores de desempeño energético, que sirva de herramienta de apoyo del control operacional y verificación de las mejoras tecnológicas que se han venido implementando en la compañía desde hace varios años.

El desarrollo e implementación de un Software de Seguimiento, Medición y Análisis del desempeño energético para cada USE's instalado, en especial del vapor generado de proceso, configurando su funcionamiento con la arquitectura informática actual de la compañía, permitirá garantizar la mejora del uso de la energía y la sostenibilidad de la reducción de costos energéticos en la empresa con medidas de baja inversión.

Se determinaron los potenciales de ahorro por consumo energético asociado y no asociado a la producción para el proceso de producción de fertilizantes, a partir de los diagramas energético productivo, la matriz energética y el análisis de los indicadores de desempeño energético, los gráficos de límites de control de consumo eléctrico, el análisis de consumo energético y producción a través del tiempo, el comportamiento de la energía en función de la producción y el Índice de consumo teórico vs Índice de consumo real.

\section{REFERENCIAS BIBLIOGRÁFICAS}

Campos, J. C., \& Prias, O. F. (2013). Implementación De Un Sistema De Gestión De La Energía, Guía Con Base En La Norma Iso 50001. Universidad Nacional de Colombia.

Dąbrowska, K. (2018). Project of the implementation of the Environmental Management System based on the standard 
ISO 14001: 2015 at Formiplast. Instytut Organizacji Systemów Produkcyjnych.

Dzene, I., Polikarpova, I., Zogla, L., \& Rosa, M. (2015). Application of ISO 50001 for Implementation of Sustainable Energy Action Plans. Energy Procedia, 72, 111-118. https://doi.org/10.1016/j. egypro.2015.06.016

Finnerty, N., Sterling, R., Coakley, D., \& Keane, M. M. (2018). An energy management maturity model for multi-site industrial organisations with a global presence. Journal of Cleaner Production, 167, 1232-1250. https://doi. org/10.1016/j.jclepro.2017.07.192

Gordić, D., Babić, M., Jovičić, N., Šušteršič, V., Končalović, D., \& Jelić, D. (2010). Development of energy management system-Case study of Serbian car manufacturer. Energy Conversion and Management, 51(12), 2783-2790.

Iwata, N. (2006). World Trade Organization and the Recycling Trade: Trade Measures for Global Environmental Preservation. Japanese Economy, 33(4), 45-64. https:// doi.org/10.2753/JES1097-203X330403

Jovanović, B., \& Filipović, J. (2016). ISO 50001 standard-based energy management maturity model - Proposal and validation in industry. Journal of Cleaner Production, 112, 2744-2755. https://doi.org/10.1016/j. jclepro.2015.10.023

Kanneganti, H., Gopalakrishnan, B., Crowe, E., Al-Shebeeb, O., Yelamanchi, T., Nimbarte, A., Currie, K., \& Abolhassani, A. (2017). Specification of energy assessment methodologies to satisfy ISO 50001 energy management standard. Sustainable Energy Technologies and Assessments, 23(June), 121-135. https:// doi.org/10.1016/j.seta.2017.09.003
Kibria, A., Akhundjanov, S. B., \& Oladi, R. (2019). Fossil fuel share in the energy mix and economic growth. International Review of Economics and Finance, 59(September 2018), 253-264. https://doi.org/10.1016/j. iref.2018.09.002

McKane, A., Therkelsen, P., Scodel, A., Rao, P., Aghajanzadeh, A., Hirzel, S., Zhang, R., Prem, R., Fossa, A., Lazarevska, A. M., Matteini, M., Schreck, B., Allard, F., Villegal Alcántar, N., Steyn, K., Hürdoğan, E., Björkman, T., \& O'Sullivan, J. (2017). Predicting the quantifiable impacts of ISO 50001 on climate change mitigation. Energy Policy, 107(May), 278-288. https:// doi.org/10.1016/j.enpol.2017.04.049

Pardo Martínez, C. I., \& Alfonso Piña, W. H. (2016). Regional analysis across Colombian departments: A non-parametric study of energy use. Journal of Cleaner Production, 115, 130-138. https://doi. org/10.1016/j.jclepro.2015.12.019

Ríos-Ocampo, J. P., Álvarez-Espinosa, A. C., Kober, T., Turner, S. W. D., Daenzer, K., Arango-Aramburo, S., Hejazi, M. I., Romero-Otalora, G. D., \& van der Zwaan, B. (2019). Climate impacts on hydropower in Colombia: A multi-model assessment of power sector adaptation pathways. Energy Policy, 128(January), 179-188. https://doi. org/10.1016/j.enpol.2018.12.057

Ylipulli, J., Suopajärvi, T., Ojala, T., Kostakos, V., Kukka, H., van den Berg, M., WendelVos, W., van Poppel, M., Kemper, H., van Mechelen, W., Maas, J., \& Corpoema, C. E. (2014). Formulación de un plan de desarrollo para las fuentes no convencionales de energia en Colombia (PDFFNCE). Technological Forecasting and Social Change, 89, 145-160. https:// doi.org/10.1016/j.techfore.2013.08.037 
